Children's Hospital, MO. Strength was determined using the British Medical Research Council Scales: $0=$ no contraction, $1=$ trace contraction, $2=$ active movement with gravity eliminated, 3 = active movement against gravity, $4=$ active movement against gravity and resistance, $5=$ normal. Range of motion and developmental skills were also assessed monthly up to 6 months, and then every 3 to 6 months, until complete recovery or absence of further improvement to age 2 years. Therapy was conducted at home and at the center.

The serial clinical examinations clearly differentiated patient outcomes: 53 (66\%) showed complete recovery, 9 (11\%) had mild weakness (4/5 strength), 7 (9\%) moderate weakness (3/5 strength), and 11 (14\%) severe weakness (0-2/5 strength). The permanently disabled infants were followed for a mean of 4.4 years. All of the infants with complete recovery had developed antigravity strength in biceps, triceps, and deltoid muscles by 4.5 months of age, and the majority by 3 months. Distal weakness resolved more rapidly, by 4 to 8 weeks. Of the 11 with severe residual disability, none had better than $2 / 5$ strength in proximal muscles at age 6 months, and $64 \%$ had $0-1 / 5$ strength in wrist and fingers. (Noetzel MJ, Park TS, Robinson S, Kaufman B. Prospective study of recovery following neonatal brachial plexus injury. $\perp$ Child Neurol July 2001;16:488-492). (Respond: Dr Michael J Noetzel, One Children's Place, Room 12E25, St Louis, MO 63110).

COMMENT. In infants with neonatal brachial plexus palsy, a detailed clinical examination with strength testing up to 6 months of age may distinguish the two-thirds who will make complete recovery from the $14 \%$ of infants left with a permanent severe disability. The early identification of those with a poor outcome may help in selection of patients for surgery at or after 6 months. In some studies, recovery has continued up to 9 months, and surgical intervention at an earlier age would be advised only rarely, with total plexus lesions.

The outcome in this study is at variance with that of 149 patients followed and treated conservatively at the Children's National Medical Center, Washington, DC. (Eng GD et al. 1996; see Progress in Pediatric Neurology III, 1997;357-9). Only 6 (4\%) showed complete recovery, and 92 (62\%) had mild impairments of strength and function. The initial clinical impairment ratings at $<3$ months of age correlated closely with later exams and with serial electrodiagnostic studies. Selection criteria for surgery were not clearly defined.

Strombeck $\mathrm{C}$ et al, at Stockholm, recommend that surgery for OBP should be delayed until after 6 to 9 months. They examined the functional outcome at age 5 years of 247 children with OBP, with or without microsurgical reconstruction. The only benefit of operation was an improved shoulder range of movement, when compared to non-operated patients. Outcome was not correlated with timing of operation, before or after 6 months. ( see Ped Neur Briefs April 2000;14:25-26).

\title{
PREDICTORS OF OUTCOME IN GUILLAIN-BARRE SYNDROME
}

Clinical and electrophysiologic predictors of outcome in 27 children with Guillain-Barre syndrome were determined by a retrospective review of records at the University of Iowa Hospitals and Clinics, lowa City, IA. Patients were divided into 2 groups; Group 1, recovery (complete or partial) after more than 2 months, and group 2, within 2 months of onset. Age at presentation ranged from 1 to 16 years (mean 9 years). Males outnumbered females 2:1. Antecendent infection (within 1 month of onset) occurred in 70\%; cytomegalovirus infection was present in $26 \%$. Numbness was the the most common presenting symptom, occurring in $48 \%$. Cranial nerve involvement in $70 \%$ (mainly VII (55\%), some multiple (29\%)) occurred especially in group 1. Intubation was required in $36 \%$ of 
group 1 only. Intravenous immunoglobulin had been used more frequently in the early recovery group 2 than group 1 patients. Features significantly different between the 2 groups were as follows: 1) maximum disability score at presentation; 2) intubation; 3) cranial nerve involvement, and 4) nerve conduction block. The maximum disability score at presentation and the probability of intubation were also significantly correlated. Features not significantly different in the 2 groups included: age, gender, preceding infection, cytomegalovirus, severity of presenting weakness, csf protein level, nerve conduction velocities and muscle and nerve action potential amplitudes, fibrillation potentials, F wave, insertional activity, axonopathy or demyelinating patterns, and IV immunoglobulin therapy. (Ammache Z, Afifi AK, Brown CK, Kimura J. Childhood Guillain-Barre syndrome: clinical and electrophysiologic features predictive of outcome. I Clin Neurol July 2001;16:477-483). (Respond: Dr Zakaria Ammache, Department of Neurology, University of lowa Hospitals and Clinics, lowa City, IA 52242).

COMMENT. Predictors of poor outcome and delayed recovery in childhood Guillain-Barre syndrome include maximum disability score at presentation, intubation, cranial nerve involvement, and conduction block. Other electrophysiological findings are not of predictive value in children. The axonal form of neuropathy, sometimes associated with a poor prognosis in adults, was present only in 2 children of the present series, both in group 1 with a delayed recovery. The difference was not significant. Campylobacter jejuni infection has been linked to a poor prognosis of GBS in 5 reports in the literature, but was not isolated in the above patient series. (See Progress in Pediatric Neurology III, 1997;pp353-4, for reports of GBS associated with C. jejuni infection).

Atypical presentation of Guillain-Barre syndrome. Eight children with GBS presented with CNS symptoms, including drowsiness, headache, and meningismus, suggesting a meningoencephalitis. (Bradshaw DY, Jones HR. Pseudomeningoencephalitic presentation of pediatric Guillain-Barre syndrome. I Child Neurol July 2001;16:505-508). Prominent CNS symptoms at onset of GBS should be recognized as part of the syndrome to avoid delay in diagnosis and treatment.

\section{ATTENTION DEFICIT DISORDERS}

\section{STMPTOMS OF ADHID IN UNIVERSTTY STUDENTS}

A sample of 1,209 university students from 3 countries (Italy, New Zealand, and USA) completed a 24-item self-report measure for ADHD symptoms in a study at Lehigh University, Bethlehem, PA. Factor analyses supported a diagnosis of ADHD, according to the bidimensional classification of DSM-IV, in the US and New Zealand samples, but less in the Italian students. The percentages of self-reported ADHD subtypes in US, Italian, and NZ students were as follows: In men, inattentive, $6,7.7$, and 8.1 , respectively; $H / I, 14,18.5$, and $10.8 ;$ Combined, $7.4,11.1,10.8$. In women, inattentive, 3.9, 9.0, and 9.8; H/I, 13.8, 25.8, 18.4; Combined, 6.9, 8.3, and 9.2, respectively. Italian students reported more inattention and $\mathrm{H} / \mathrm{I}$ than US students. NZ students had significantly more inattentive symptoms than US students, especially women. (DuPaul GJ, Schaughency EA, Weyandt LL et al. Self-report of ADHD symptoms in university students: cross-gender and cross-national prevalence. L Learning Disabilities July/Aug 2001;34:370-379). (Respond: G J DuPaul, School Psychology Program, Lehigh Univ, 111 Research Dr, Bethlehem, PA 18015). . 